\title{
Flies, cockroaches and AMR
}

\author{
Epidemiological surveillance highlights a role for flies, spiders and cockroaches in the antimicrobial-resistance \\ pandemic.
}

$\mathrm{R}$ esistance of microorganisms to antimicrobials (antibiotics, antivirals, antifungals or antiparasitics), or AMR as it is more commonly known, poses an enormous threat to human and veterinary medicine. This is because AMR could render many of the current mainstay and last-resort antibiotics useless, which would result in deaths from previously treatable infections. As the problem of AMR grows relentlessly year on year, the number of effective antimicrobials is reduced. AMR is clearly a problem for the treatment of infectious disease, but like ripples on a pond, AMR adversely affects progress towards several of the UN's Sustainable Development Goals, including those addressing poverty (inextricably linked with disease), food production and clean water for all. Refilling the antimicrobial pipeline is one answer, but AMR is currently outpacing antimicrobial drug development, so there is political will to tackle the problem. The question is how.

Overcoming AMR is a complex endeavour that necessitates integration of different areas of expertise - infectious disease, molecular biology, synthetic chemistry, biochemistry, human-animalenvironmental epidemiology, clinical, modelling, health and social policy, and more - to understand the problem and to enable the provision of robust solutions. In 2017, the World Health Organization (WHO), the Food and Agriculture Organization and the World Organisation for Animal Health, also known as 'The Tripartite, advocated for the adoption of a One Health approach to addressing AMR. In July 2020, the WHO issued a call to recruit leaders in different sectors from around the world to form a group focused on tackling AMR, and in January 2021, the inaugural meeting of this WHO-convened Global Leaders Group on Antimicrobial Resistance (https://www.who.int/groups/ one-health-global-leaders-group-onantimicrobial-resistance) was held. The visions and action plan were articulated in a report on the Inaugural Meeting 26-27 January 2021 (ref. $^{1}$ ) and included a focus on the necessity to make the biggest impact on combating AMR in low- and middle-income countries (LMICs), where the need is most acute, and to identify the root causes of AMR to enable the provision of sustainable solutions.
AMR is a One Health ${ }^{2}$ problem. Resistance can spread within hospitals, communities, farms and wastewater systems. We already know that domestic and companion animals ${ }^{3}$ share AMR microorganisms with humans. We also know that antibiotic misuse provides a selection pressure that enables transmission networks of AMR organisms to expand. But what about the role of potential vectors such as insects and spiders in AMR? A growing body of evidence published over the past six years has shown that flies and cockroaches on farms can harbour AMR microorganisms, but little attention has been paid to arthropod populations in hospitals. In South Asia and other LMICs, insect populations are rarely well-controlled in healthcare settings and, moreover, have open access to many patient wards. Whether this has any bearing on AMR in LMICs has remained an open question. In this issue of Nature Microbiology, in their Article, Hassan et al. combine patient demographics, sample collection in two seasons and in-depth bacterial genomics analyses to provide compelling evidence of connections between antimicrobial-resistant bacteria in arthropods, in surgical-site infections and on hospital surfaces in a semi-urban South Asian hospital.

Using an observational, longitudinal study design, Hassan et al. collected ants, spiders, cockroaches and moths, and trapped flies in a hospital, and isolated AMR bacteria from these arthropods, as well as from hospital surfaces and surgical-site patient infections. They undertook a coordinated approach to characterize a holistic picture of AMR inside a typical semi-urban public hospital. Importantly, their study design included single nucleotide polymorphisms in the whole-genome sequencing analysis of the isolated multi-drug-resistant Enterobacterales to examine spatiotemporal connectivity between samples of different origins. As also seen in a study published in 2019 (ref. ${ }^{4}$ ), they detected substantial amounts of multi-drug-resistant Enterobacterales on surfaces in the hospital. The multi-drug-resistant Enterobacterales analysed by Hassan et al. included species that are resistant to carbapenem - a drug reserved for life-threatening infections. The main advance they report is that arthropods have a role in the dissemination of extremely drug-resistant Enterobacterales (XDRE) that have clonal links with clinical samples. Their study reveals that $20 \%$ of the flies, cockroaches, spiders, moths and ants they sampled carry carbapenem resistance and 70-80\% carry extended spectrum cephalosporin resistance. These striking findings suggest that insects and spiders need to be removed from healthcare environments.

Is this a surprising result? Perhaps not. That being said, it is not trivial to set up an exhaustive sampling effort over multiple weeks and seasons in a busy hospital environment. Follow-up research to devise sampling strategies and experimental designs to dissect out causation and correlation may prove vital in informing public-health policies to reduce insects and spiders in hospital and healthcare environments.

Research into detection, mechanisms, spread and countering of AMR crosses disciplines and continents alike. Sustainable country-level policies to tackle AMR will require robust country-level evidence. Publishing the very best research from those countries with the highest burden of AMR in our journal is one way that we can highlight research that may eventually underpin policies to reduce the burden of AMR.

Getting research findings relevant to the spread of AMR translated into public-health policies will present a different set of challenges. In a 2019 policy briefing from the WHO that examined the centrality of culture to the challenge of antibiotic resistance ${ }^{5}$, the authors noted that "Translation of high-income country (HIC) policies to low-and middle-income countries (LMICs) will not be effective if the contexts of medicine use and the specificities of settings are not taken into account." Moving from research that identifies potential solutions, such as control of insects and spiders to reduce the chance of acquiring a multi-drug-resistant pathogen by a patient, to policies will be key to advancing the AMR debate from calls for action to action itself.

Translating basic research into effective public health policies to overcome AMR will take time. In the case of moving insects harbouring AMR microorganisms, there are a slew of methods available to reduce or exclude insects from hospitals, including identifying and removing breeding sites, 
ensuring buildings have doors and windows that keep flies out, ultraviolet light traps and pesticides. Local budgets and the acceptability of potential solutions will need to dovetail with the most appropriate measure(s). As well as local research evidence and inclusion of country-level civil servants, clinicians, scientists and community leaders to identify suitable solutions, all policies will require testing. In June 2020, a framework for setting up and testing intervention for AMR was reported ${ }^{6}$, which should help those who turn basic research into effective public health policies.

By highlighting AMR research carried out in both HICs and LMICs in our journal, we hope to contribute to efforts to develop sustainable country-level policies that can extend the lifetime of the precious antimicrobials that we have come to depend on so much.

Published online: 27 September 2021 https://doi.org/10.1038/s41564-021-00977-x
References

1. Final Report of the Inaugural Online Meeting of the Global Leaders Group on Antimicrobial Resistance. January 26-27, 2021 (Global Leaders Group on Antimicrobial Resistance, 2021).

2. One Health https://www.who.int/news-room/q-a-detail/ one-health (WHO, 2017).

3. Li, J. et al. Environ. Health Perspect. 127, 107009 (2019).

4. D'Souza, A. W. Nat. Commun. 10, 4569 (2019).

5. Ledingham, K., Hinchcliffe, S., Jackson, M., Thomas, F. \& Tomson, G. Antibiotic Resistance: Using a Cultural Contexts of Health Approach to Address a Global Health Challenge (WHO, 2019).

6. Rogers Van Katwyk, S., Hoffman, S. J., Mendelson, M., Taljaard, M. \& Grimshaw, J. M. Health Res. Policy Syst. 18 60 (2020). 